\title{
Electron-Deficient $\beta$-Diiminato-Zinc-Ethyl Complexes: Synthesis, Structure and Reactivity in Ring-Opening Polymerization of Lactones
}

\begin{abstract}
Alexander Kronast ${ }^{\dagger, \neq}$, Marina Reiter ${ }^{\dagger, \neq}$, Peter T. Altenbuchner ${ }^{\dagger}$, Christian Jandl ${ }^{\#}$, Alexander Pöthig ${ }^{\#}$, and Bernhard Rieger ${ }^{+*}$.

${ }^{+}$WACKER-Lehrstuhl für Makromolekulare Chemie, Technische Universität München, Lichtenbergstraße 4, 85747 Garching bei München, Germany

\#Zentralinstitut für Katalyseforschung, Technische Universität München, Ernst-Otto-Fischer Straße 1, 85747 Garching bei München, Germany
\end{abstract}

\section{Supporting Information}

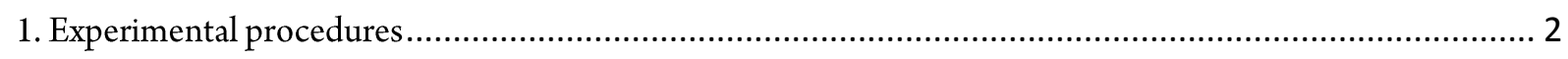

Methods and materials.................................................................................................. 2

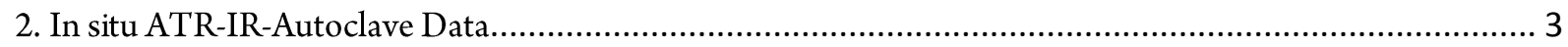

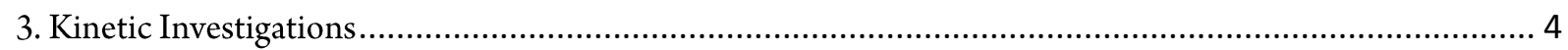

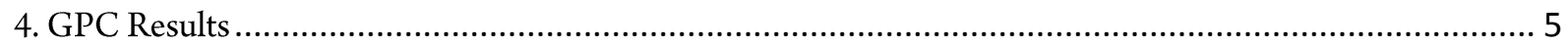

5. Microstructure determination via NMR Spectroscopy …........................................................ 9

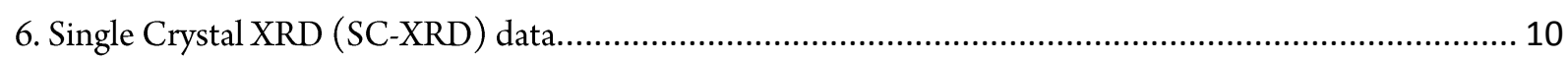




\section{EXPERIMENTAL PROCEDURES}

\section{METHODS AND MATERIALS}

Unless otherwise stated, all manipulations were performed under an argon atmosphere using standard Schlenk techniques or an MBraun glovebox. Chemicals were purchased from Sigma-Aldrich or ABCR GmbH\&Co KG and used without further treatment if not otherwise stated. All glassware was heat-dried under vacuum prior to use. Toluene, dichloromethane, diethylether, pentane and tetrahydrofuran were dried applying an MBraun SPS800 and used as received. Monomers were dried over calcium hydride and distilled prior to polymerization. NMR spectra were recorded on a Bruker AVIII-300 and AVIII-500 Cryo spectrometer. ${ }^{1} \mathrm{H}$ NMR spectroscopic chemical shifts $\delta$ are reported in ppm relative to tetramethylsilane and calibrated to the residual proton signal of the deuterated solvent. Deuterated solvents were obtained from Sigma Aldrich and dried over 3 A molecular sieves. ESI-MS analytical measurements were performed in acetonitrile solutions on a Varian 500 MS spectrometer. In-situ IR measurements were carried out under an argon atmosphere using an ATR-IR Mettler Toledo system. IR spectra were performed on a Bruker Vertex 70 spectrometer with a Bruker Platinum ATR setup and the integrated MCT detector. Gel permeation chromatography (GPC) analysis was performed on a Varian PL-GPC 50. As eluent, chloroform (HPLC grade) with $1.5 \mathrm{~g} \mathrm{~L}^{-1}$ tetrabutylammonium tetrafluoroborate was used. Polystyrene standards were used for calibration. 


\section{IN SITU ATR-IR-AUTOCLAVE DATA}

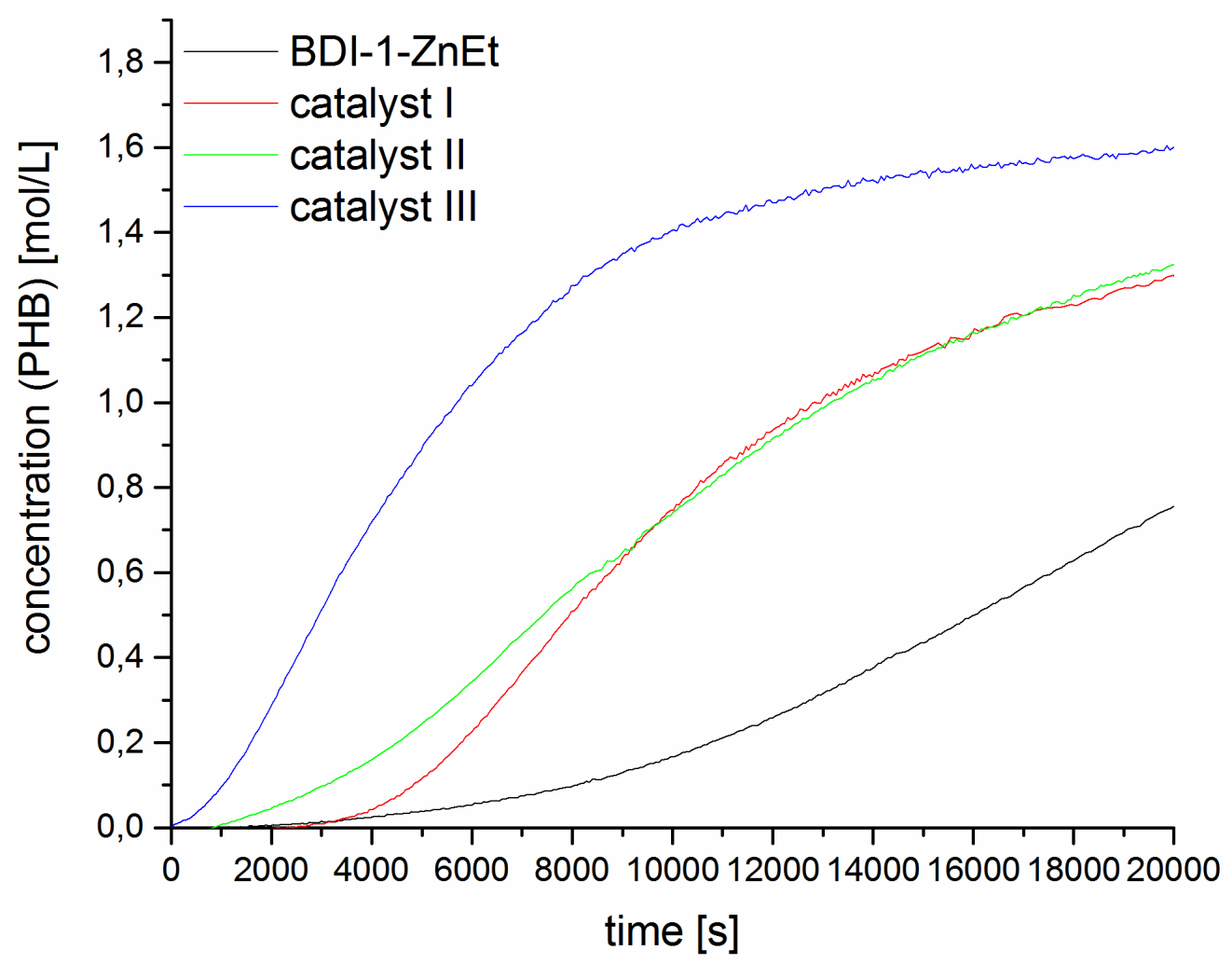

Figure S1. In situ IR data for the ROP of rac-BL with literature known catalyst BDI-1-ZnEt and complexes I-III during the first $5.5 \mathrm{~h}$. 


\section{KinETIC INVESTIGATIONS}
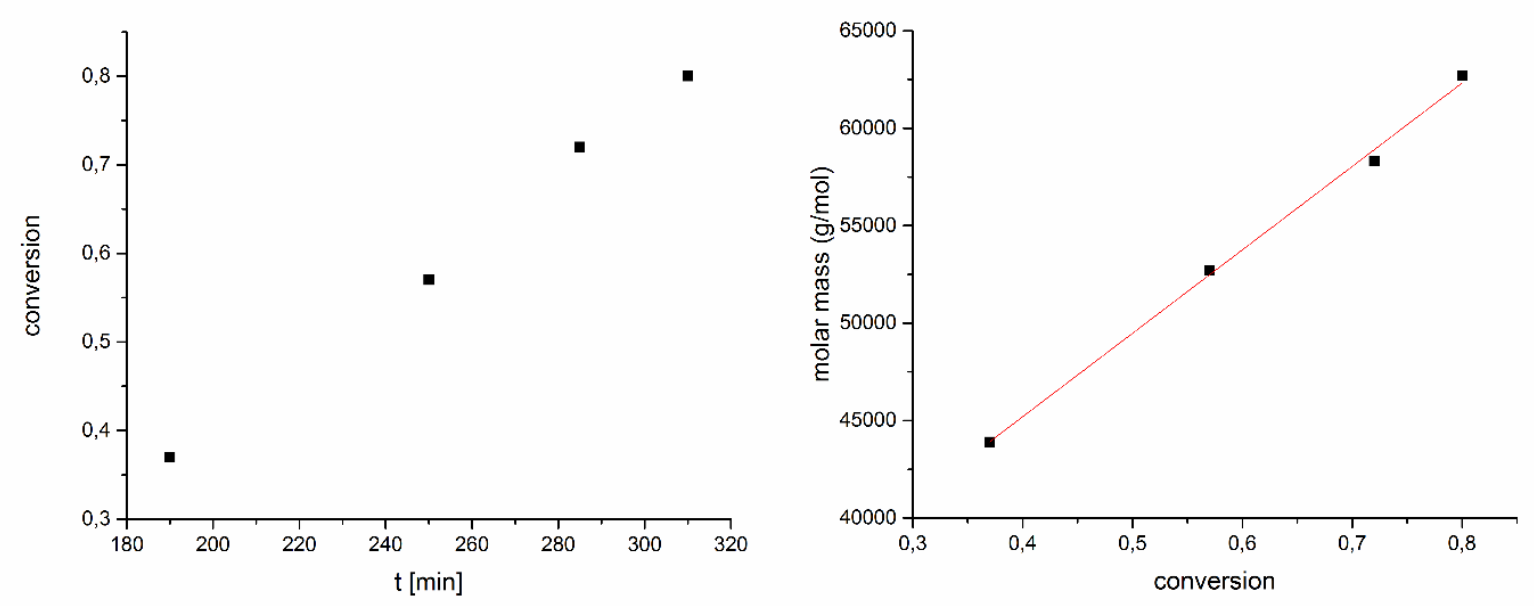

Figure S2. Catalytic activity of III for the ROP of rac-BL (left) and linear growth of $\mathrm{M}_{\mathrm{n}}$ as a function of monomer conversion (determined by ${ }^{1} \mathrm{H} \mathrm{NMR}$ ) (right). 


\section{GPC RESULTS}

a) Polyhydroxybutyrate:

\section{BDI-1-ZnEt}

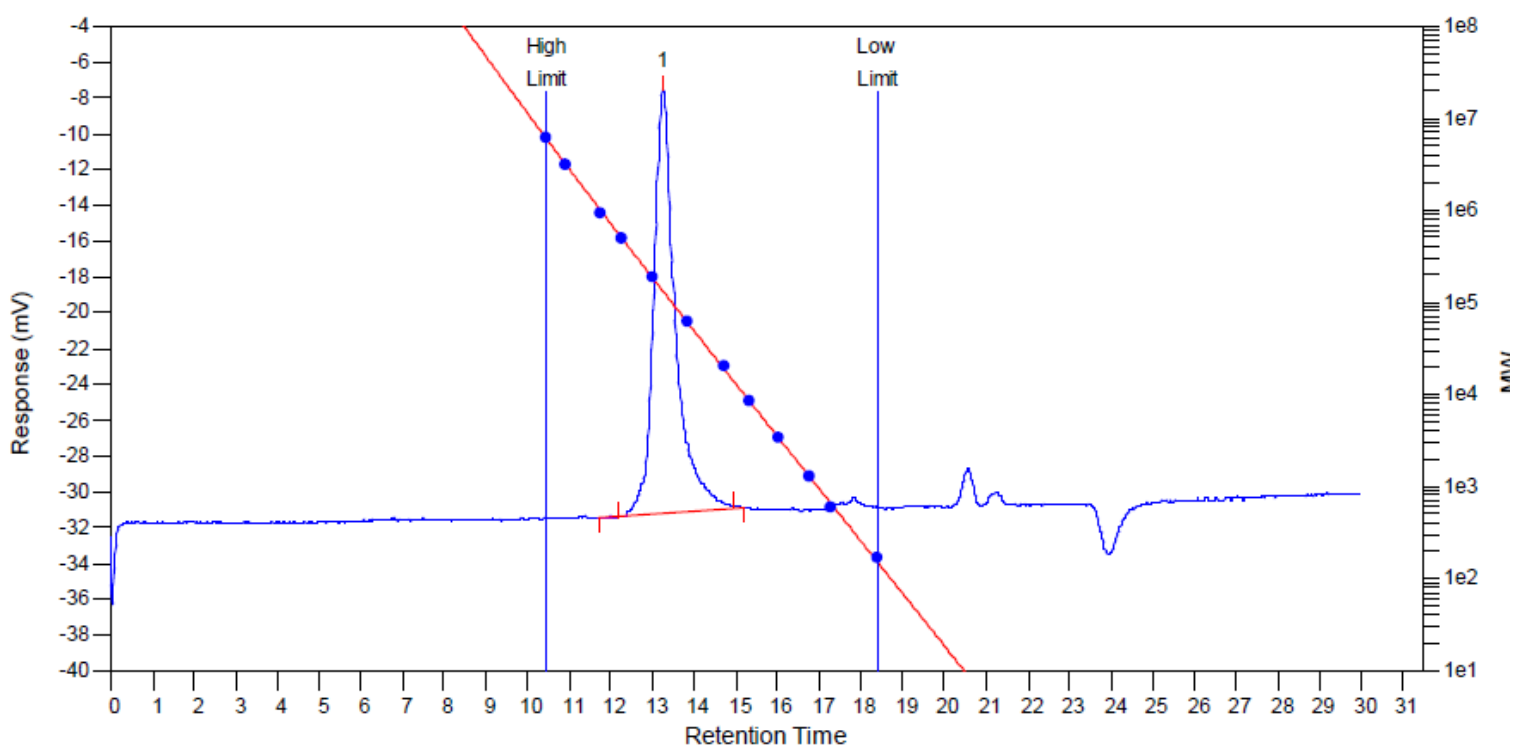

MW Averages

$\begin{array}{rccccccc}\text { Peak No } & \mathrm{Mp} & \mathrm{Mn} & \mathrm{Mw} & \mathrm{Mz} & \mathrm{Mz}+1 & \mathrm{Mv} & \mathrm{PD} \\ 1 & 130967 & 99335 & 124644 & 145798 & 167933 & 121430 & 1.25478\end{array}$

\section{Catalyst I}

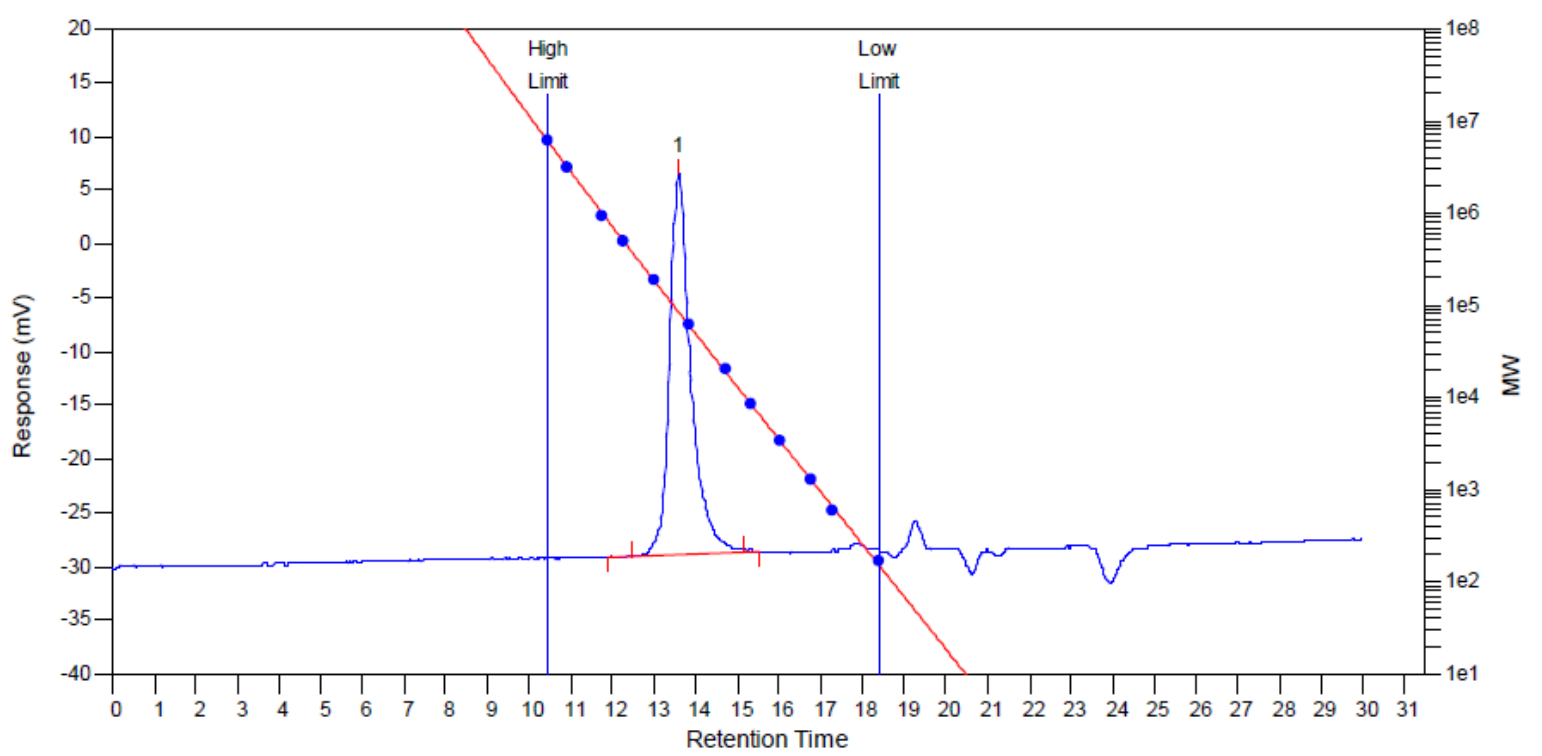

MW Averages

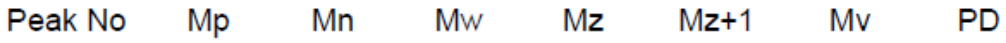

$\begin{array}{llllllll}1 & 83861 & 66613 & 79436 & 90772 & 102784 & 77732 & 1.1925\end{array}$




\section{Catalyst II}

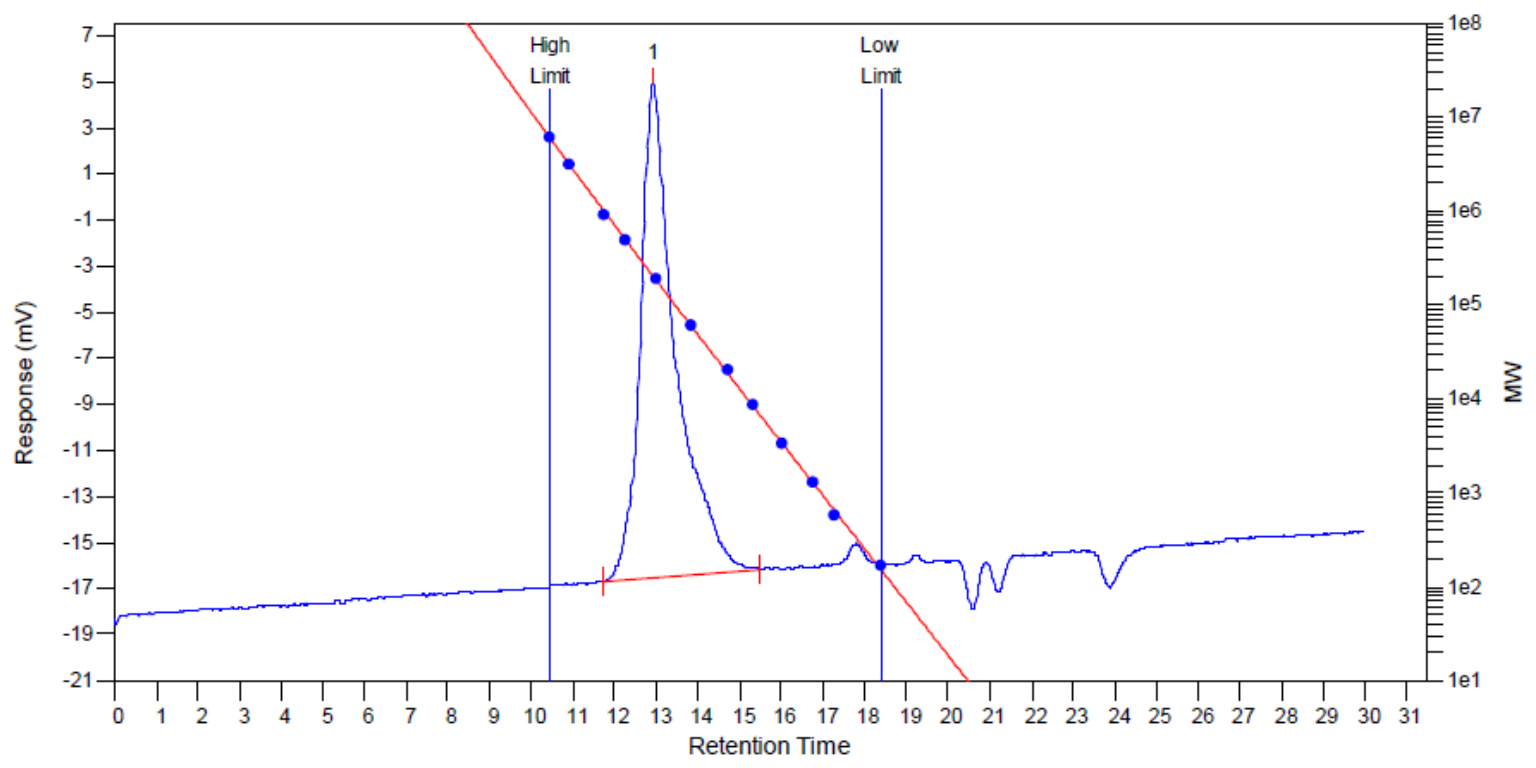

MW Averages

$\begin{array}{llllllll}\text { Peak No } & M p & M n & M w & M z & M z+1 & M v & \text { PD }\end{array}$

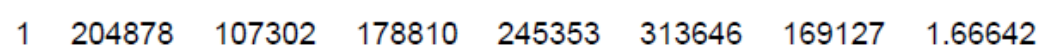

\section{Catalyst III}

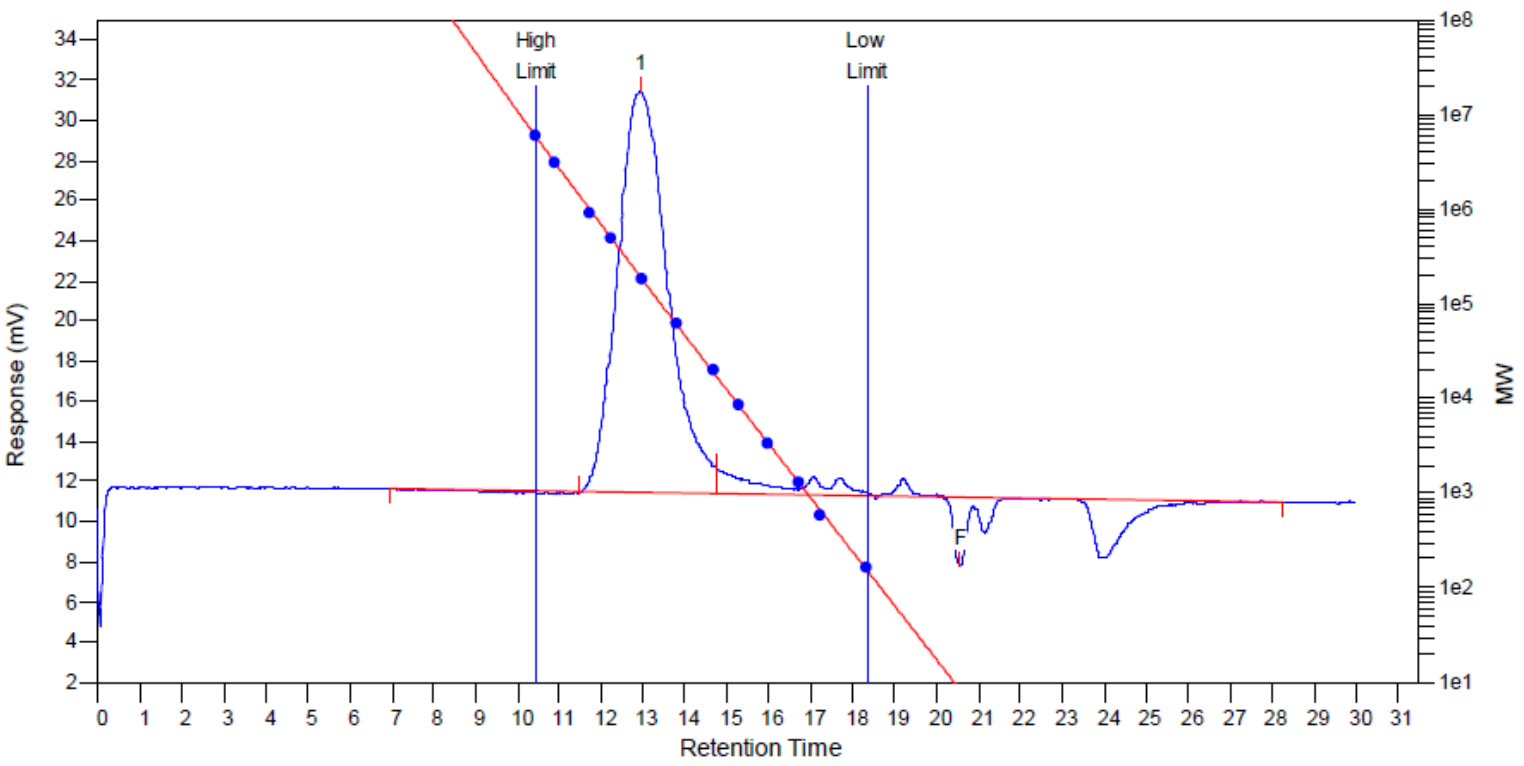

MW Averages

$\begin{array}{rcrrrrrr}\text { Peak No } & \text { Mp } & \text { Mn } & \text { Mw } & \text { Mz } & \text { Mz+1 } & \text { Mv } & \text { PD } \\ 1 & 196402 & 120787 & 214077 & 331085 & 456979 & 198757 & 1.77235 \\ 2 & 0 & 0 & 0 & 0 & 0 & 0 & 0\end{array}$ 


\section{b) Polylactide}

\section{BDI-1-ZnEt}

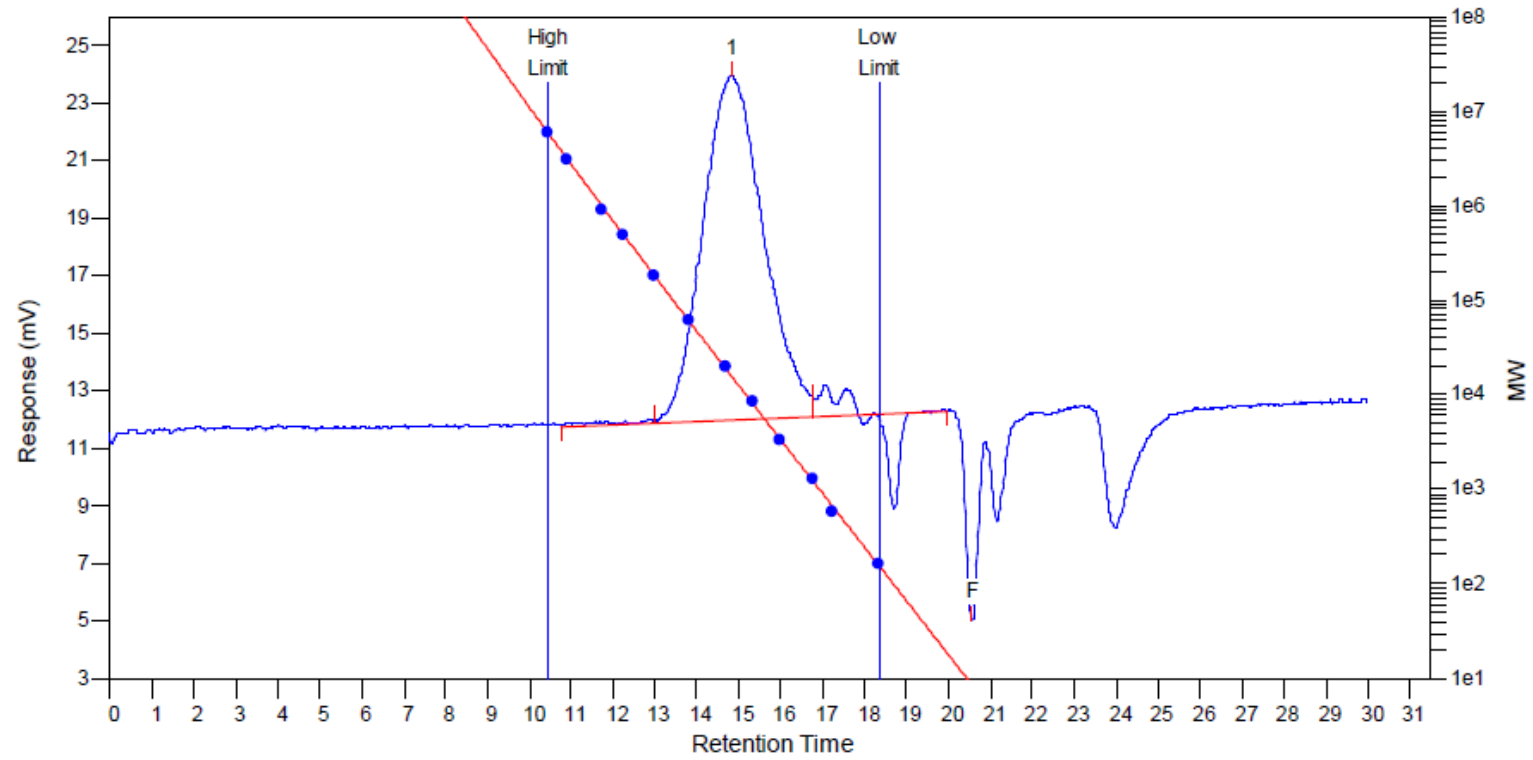

MW Averages

$\begin{array}{rcrccccc}\text { Peak No } & \text { Mp } & \text { Mn } & \text { Mw } & \text { Mz } & M z+1 & \text { Mv } & \text { PD } \\ 1 & 15812 & 9344 & 20041 & 36990 & 58197 & 18074 & 2.1448 \\ 2 & 0 & 0 & 0 & 0 & 0 & 0 & 0\end{array}$

\section{Catalyst II}

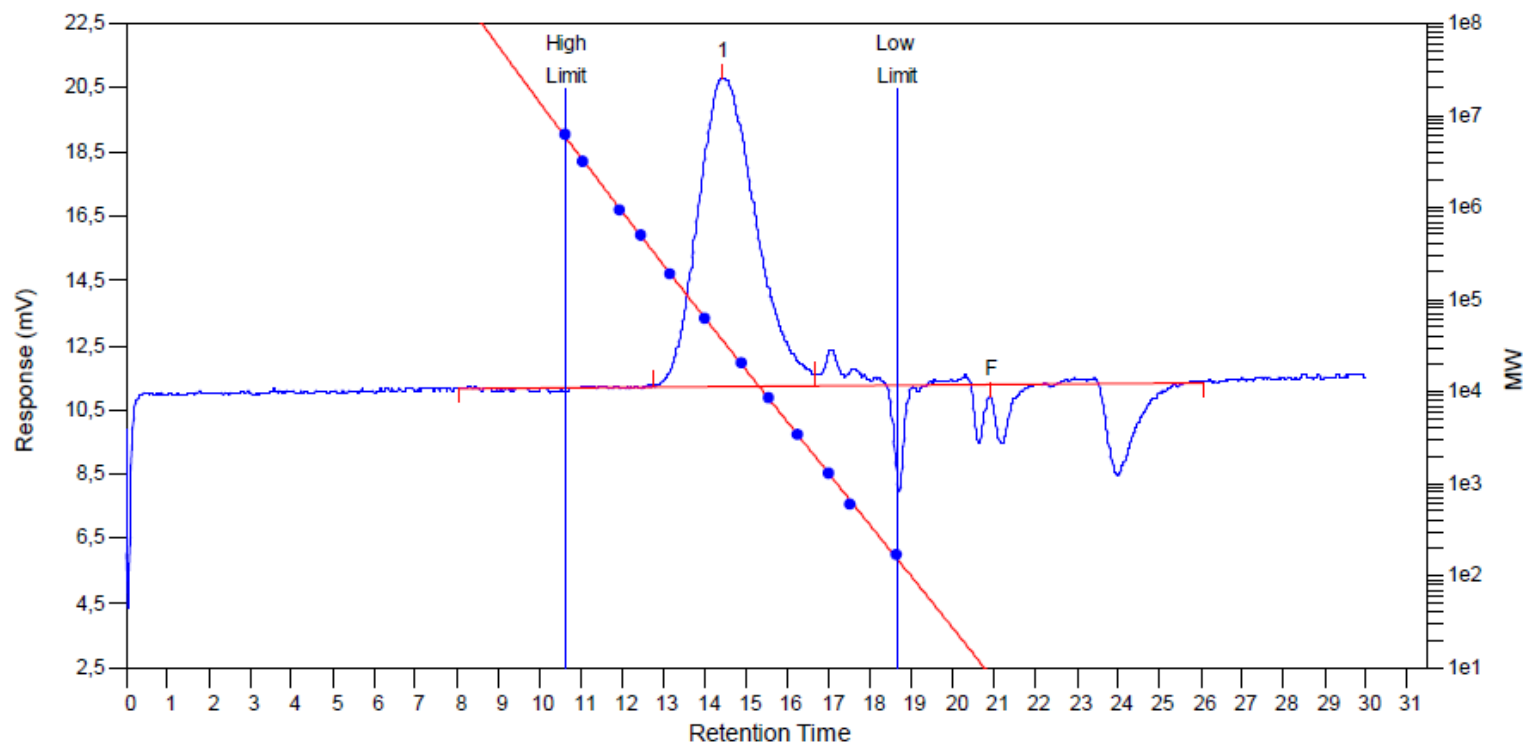

MW Averages

$\begin{array}{rcrrccrr}\text { Peak No } & \mathrm{Mp} & \mathrm{Mn} & \mathrm{Mw} & \mathrm{Mz} & \mathrm{Mz}+1 & \mathrm{Mv} & \mathrm{PD} \\ 1 & 34884 & 18764 & 39500 & 67902 & 100507 & 35980 & 2.10509 \\ 2 & 0 & 0 & 0 & 0 & 0 & 0 & 0\end{array}$




\section{Catalyst III}

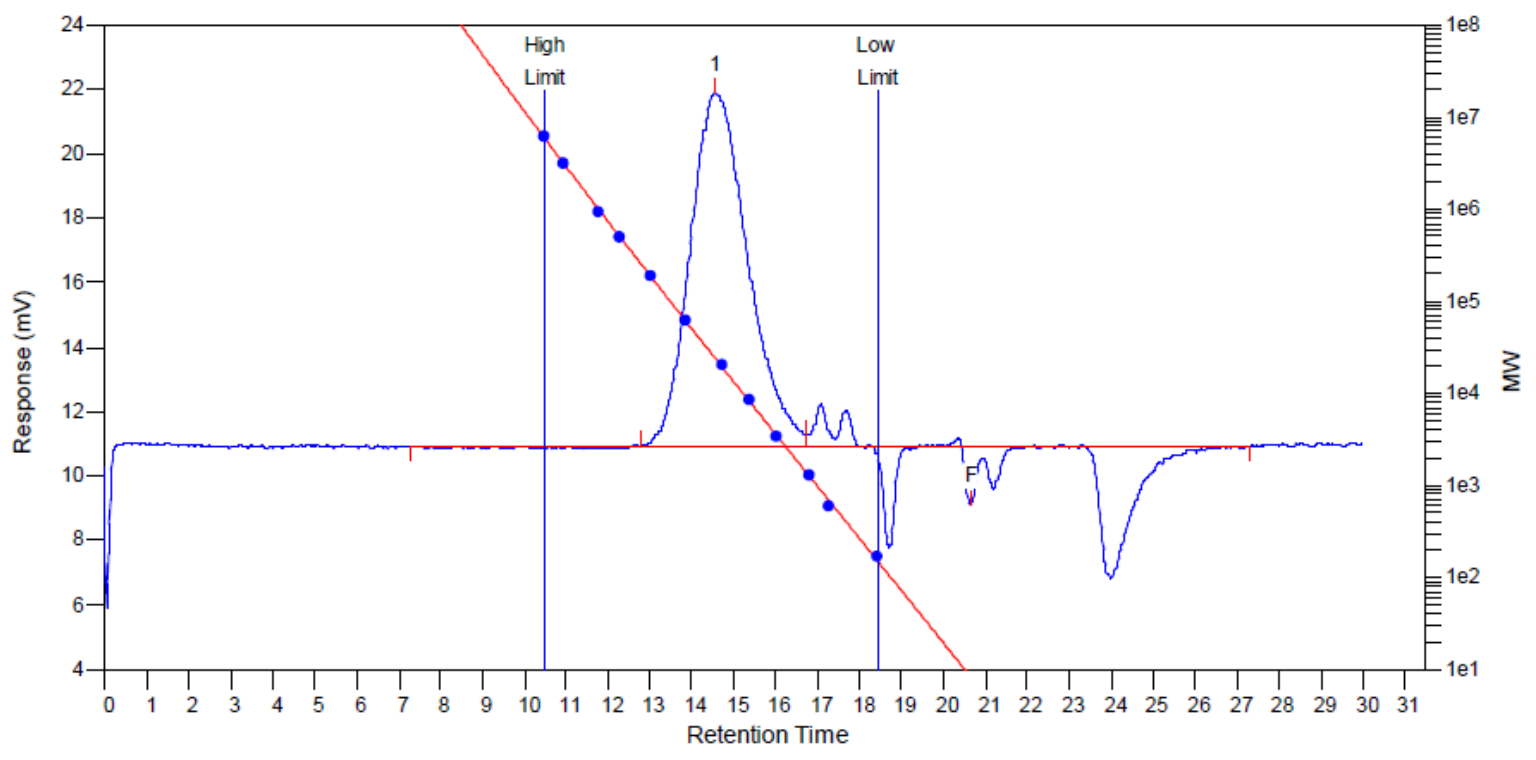

MW Averages

$\begin{array}{rcrrrrrr}\text { Peak No } & \text { Mp } & \text { Mn } & \text { Mw } & \text { Mz } & M z+1 & M v & \text { PD } \\ 1 & 23949 & 13050 & 27249 & 48025 & 74372 & 24775 & 2.08805 \\ 2 & 0 & 0 & 0 & 0 & 0 & 0 & 0\end{array}$




\section{MiCROSTRUCTURE DETERMINATION VIA NMR}

\section{SPECTROSCOPY}

\section{Polyhydroxybutyrate}

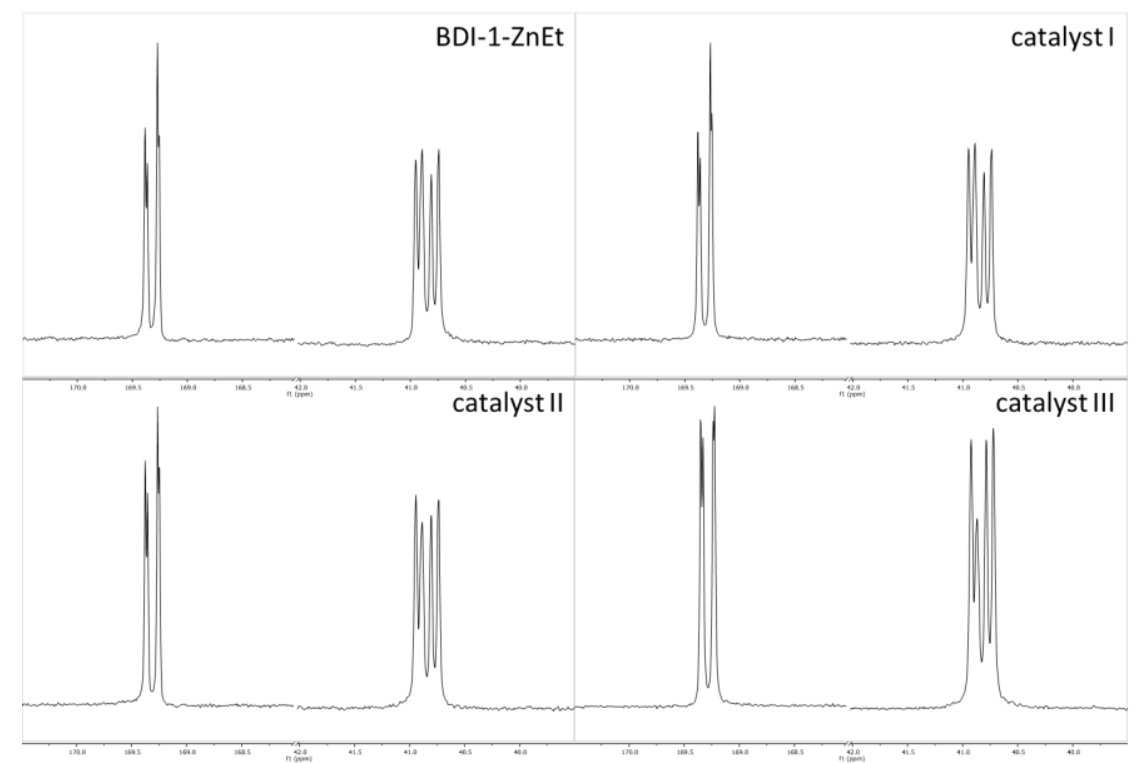

Figure S3. ${ }^{13} \mathrm{C}$ spectra applied in the microstructure analysis for polyhydroxybutyrates obtained with BDI-1-ZnEt and catalysts I-III (methylene (40.8 ppm) and carbonyl regions (169.3 ppm)).

\section{Polylactide}

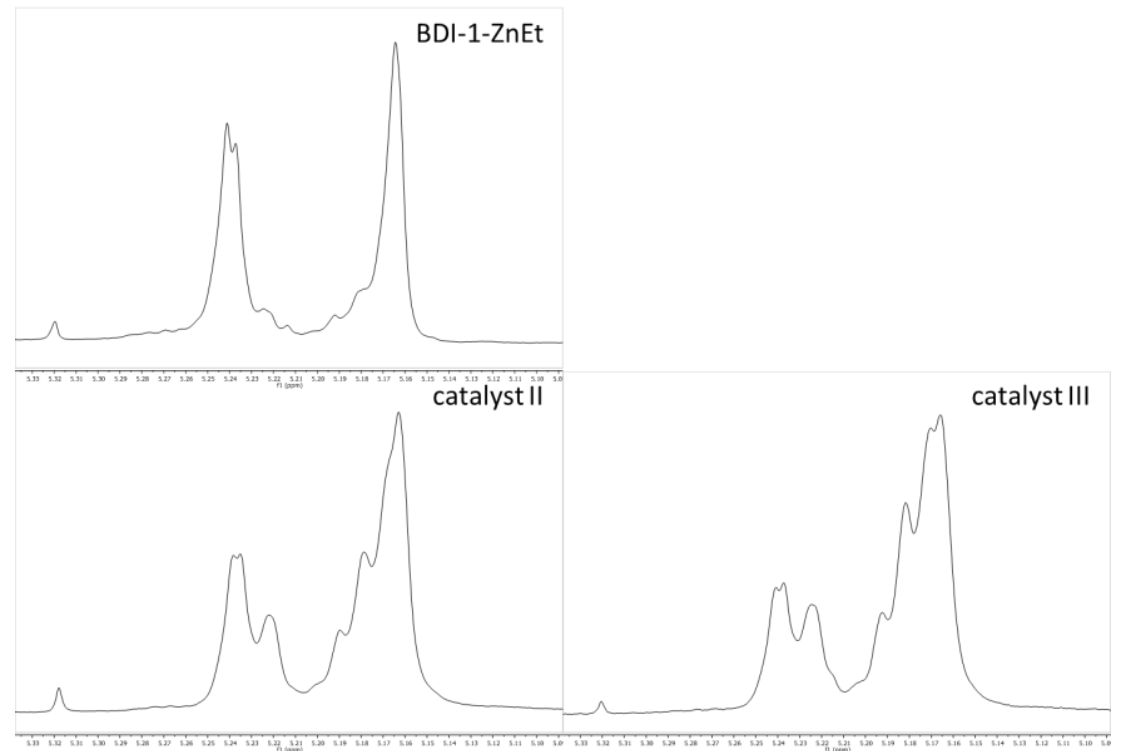

Figure S4. Homodecoupled ${ }^{1} \mathrm{H}-\mathrm{NMR}$ spectra applied in the microstructure analysis for polylactides obtained with BDI-1-ZnEt and catalysts II-III (methine region). 


\section{Single Crystal XRD (SC-XRD) data}

\section{General:}

Data were collected on an X-ray single crystal diffractometer equipped with a CCD detector (APEX II, CCD), a rotating anode FR591 and a Montel mirror optic (Compound I) or a fine-focus sealed tube and a Triumph optic (Compound II, III) by using the SMART software package. ${ }^{(1)}$ The measurements were performed on single crystals coated with perfluorinated ether. The crystals were fixed on the top of a glass fiber and transferred to the diffractometer. Crystals were cooled under a stream of cold nitrogen. A matrix scan was used to determine the initial lattice parameters. Reflections were merged and corrected for Lorentz and polarization effects, scan speed, and background using SAINT.(2) Absorption corrections, including odd and even ordered spherical harmonics were performed using SADABS. ${ }^{(2)}$ Space group assignments were based upon systematic absences, $E$ statistics, and successful refinement of the structures. Structures were solved by direct methods with the aid of successive difference Fourier maps, ${ }^{(3)}$ and were refined against all data using the APEX 2 software ${ }^{(1)}$ in conjunction with SHELXL-97 or SHELXL-2014(4) and SHELXLE ${ }^{(5)}$. Methyl hydrogen atoms were refined as part of rigid rotating groups, with a C-H distance of $0.98 \AA$ and $\mathrm{U}_{\mathrm{iso}(\mathrm{H})}=1.5 \cdot \mathrm{U}_{\mathrm{eq}(\mathrm{C})}$. Other $\mathrm{H}$ atoms were placed in calculated positions and refined using a riding model, with methylene and aromatic $\mathrm{C}-\mathrm{H}$ distances of 0.99 and $0.95 \AA$, respectively, and $\mathrm{U}_{\mathrm{iso}(\mathrm{H})}=1.2 \cdot \mathrm{U}_{\mathrm{eq}(\mathrm{C})}$. Non-hydrogen atoms were refined with anisotropic displacement parameters. Full-matrix least-squares refinements were carried out by minimizing $\Sigma w\left(\mathrm{~F}_{\mathrm{o}}^{2}-\mathrm{F}_{\mathrm{c}}^{2}\right)^{2}$ with SHELXL-97 $7^{(4)}$ weighting scheme. Neutral atom scattering factors for all atoms and anomalous dispersion corrections for the non-hydrogen atoms were taken from International Tables for Crystallography. ${ }^{(6)}$ Images of the molecular structure in the single crystal were generated with PLATON. ${ }^{(7)}$ For compound I a superstructure with a tripled $\mathrm{b}$ axis was identified but the data quality did not allow a proper refinement so the smaller higher symmetric cell was used.

\section{References:}

(1) APEX suite of crystallographic software. APEX 2 Version 2013.4. Bruker AXS Inc., Madison, Wisconsin, USA (2013).

(2) SAINT, Version 8.27b and SADABS Version 2012/1. Bruker AXS Inc., Madison, Wisconsin, USA (2012).

(3) Sheldrick, G. M. "SHELXS-97", Program for Crystal Structure Solution, Göttingen, (1997).

(4) Sheldrick, G. M. "SHELXL-97", University of Göttingen, Göttingen, Germany, (1998). or Sheldrick, G. M. "SHELXL-2014", University of Göttingen, Göttingen, Germany, (2014).

(5) Huebschle, C. B., Sheldrick, G. M. \& Dittrich, B. "SHELXLE”, J. Appl. Cryst. 2011, 44, 1281- 1284.

(6) International Tables for Crystallography, Vol. C, Tables 6.1.1.4 (pp. 500-502), 4.2.6.8 (pp. 219-222), and 4.2.4.2 (pp. 193-199), Wilson, A. J. C., Ed., Kluwer Academic Publishers, Dordrecht, The Netherlands, 1992.

(7) Spek, A. L. "PLATON", A Multipurpose Crystallographic Tool, Utrecht University, Utrecht, The Netherlands, (2011). 


\section{Compound I (CCDC 1439897)}

Diffractometer operator C. Jandl scanspeed 5 s per frame

$\mathrm{dx} 70 \mathrm{~mm}$

5762 frames measured in 15 data sets

phi-scans with delta_phi $=0.5$

omega-scans with delta_omega $=0.5$

\section{Crystal data}

\section{$\mathrm{C}_{31} \mathrm{H}_{40} \mathrm{~F}_{6} \mathrm{~N}_{2} \mathrm{Zn}$}

\begin{tabular}{l|l}
$M_{\mathrm{r}}=620.04$ & $D_{\mathrm{x}}=1.325 \mathrm{Mg} \mathrm{m}^{-3}$ \\
\hline
\end{tabular}

Monoclinic, $C 2 / \mathrm{c}$

Melting point: ? K

Hall symbol: -C 2yc

Mo Ka radiation, $\lambda=0.71073 \AA$

$a=35.2290(11) \AA$

Cell parameters from 9001 reflections

$b=11.4852(3) \AA$

$\theta=2.4-28.1^{\circ}$

$c=16.5381(5) \AA$

$\mu=0.85 \mathrm{~mm}^{-1}$

$\beta=111.691(1)^{\circ}$

$T=123 \mathrm{~K}$

$V=6217.7(3) \AA^{3}$

Fragment, yellow

$Z=8$

$0.53 \times 0.32 \times 0.24 \mathrm{~mm}$

$F(000)=2592$

\section{Data collection}

\begin{tabular}{|l|l|}
\hline $\begin{array}{l}\text { Bruker APEX-II CCD } \\
\text { diffractometer }\end{array}$ & 5686 independent reflections \\
\hline Radiation source: rotating anode FR591 & 5156 reflections with $\mathrm{i}>2 \sigma(\mathrm{i})$ \\
\hline MONTEL optic monochromator & $R_{\text {int }}=0.041$ \\
\hline Detector resolution: 16 pixels $\mathrm{mm}^{-1}$ & $\theta_{\max }=25.4^{\circ}, \theta_{\min }=1.9^{\circ}$ \\
\hline phi- and $\omega$-rotation scans & $h=-42 \quad 42$ \\
\hline $\begin{array}{l}\text { Absorption correction: multi-scan } \\
S A D A B S, \text { Bruker, } 2008 \mathrm{~b}\end{array}$ & $k=-13 \quad 13$ \\
\hline$T_{\min }=0.602, T_{\max }=0.746$ & $1=-19 \quad 19$ \\
\hline
\end{tabular}


77572 measured reflections

Refinement

Refinement on $F^{2}$

Least-squares matrix: full

$R\left[F^{2}>2 \sigma\left(F^{2}\right)\right]=0.039$

$w R\left(F^{2}\right)=0.097$

$S=1.03$

5686 reflections

446 parameters

41 restraints

- constraints

Primary atom site location: structure-invariant direct methods
Secondary atom site location: difference

Fourier map

Hydrogen site location: inferred from neighbouring sites

$\mathrm{H}$-atom parameters constrained

$\mathrm{W}=1 /\left[\Sigma^{2}\left(\mathrm{FO}^{2}\right)+(0.0399 P)^{2}+14.6952 P\right]$

WHERE $P=\left(F^{2}+2 F C^{2}\right) / 3$

$(\Delta / \sigma)_{\max }=0.001$

$\Delta \rho_{\max }=0.81$ e $\AA^{-3}$

$\Delta \rho_{\min }=-0.63$ e $\AA^{-3}$

Extinction correction: none

Extinction coefficient: - 


\section{Compound II (CCDC 1439898)}

Diffractometer operator C. Jandl scanspeed 1 s perframe $\mathrm{dx} 40 \mathrm{~mm}$ 3804 frames measured in 10 data sets phi-scans with delta_phi $=0.5$ omega-scans with delta_omega $=0.5$

\section{Crystal data}

\begin{tabular}{|l|l|}
\hline $\mathrm{C}_{27} \mathrm{H}_{32} \mathrm{~F}_{6} \mathrm{~N}_{2} \mathrm{Zn}$ & $D_{\mathrm{x}}=1.395 \mathrm{Mg} \mathrm{m}^{-3}$ \\
\hline$M_{\mathrm{r}}=563.94$ & Melting point: ? K \\
\hline Monoclinic, $P 2_{1} / n$ & Mo Ka radiation, $\lambda=0.71073 \AA$ \\
\hline Hall symbol: -P 2yn & Cell parameters from 9804 reflections \\
\hline$a=12.4913(7) \AA$ & $\theta=2.5-26.3^{\circ}$ \\
\hline$b=10.7240(6) \AA$ & $\mu=0.97 \mathrm{~mm}^{-1}$ \\
\hline$c=20.1647(11) \AA$ & $T=150 \mathrm{~K}$ \\
\hline$\beta=96.137(3)^{\circ}$ & Fragment, yellow \\
\hline$V=2685.7(3) \AA^{3}$ & $0.53 \times 0.44 \times 0.34 \mathrm{~mm}$ \\
\hline$Z=4$ & \\
\hline$F(000)=1168$ & \\
\hline
\end{tabular}

Data collection

\begin{tabular}{|l|l|}
\hline $\begin{array}{l}\text { Bruker APEX-II CCD } \\
\text { diffractometer }\end{array}$ & 5299 independent reflections \\
\hline $\begin{array}{l}\text { Radiation source: fine-focus sealed tube } \\
4766 \text { reflections with i }>2 \sigma(\mathrm{i})\end{array}$ \\
\hline $\begin{array}{l}\text { Triumph optic monochromator } \\
\text { Detector resolution: } 16 \text { pixels } \mathrm{mm}^{-1}\end{array}$ & $\theta_{\text {int }}=0.023$ \\
\hline phi-and $\omega$-rotation scans & $h=-15 \quad 15$ \\
\hline $\begin{array}{l}\text { Absorption correction: multi-scan } \\
S A D A B S, \text { Bruker, } 2008 \mathrm{~b}\end{array}$ & $k=-13 \quad 13$ \\
\hline$T_{\min }=0.685, T_{\max }=0.745$ & $1=-24 \quad 24$ \\
\hline
\end{tabular}


78871 measured reflections

Refinement

Refinement on $F^{2}$

Least-squares matrix: full

$R\left[F^{2}>2 \sigma\left(F^{2}\right)\right]=0.028$

$w R\left(F^{2}\right)=0.077$

$S=1.06$

5299 reflections

330 parameters

0 restraints

- constraints

Primary atom site location: structure-invariant direct methods
Secondary atom site location: difference

Fourier map

Hydrogen site location: inferred from neighbouring sites

$\mathrm{H}$-atom parameters constrained

$\mathrm{W}=1 /\left[\Sigma^{2}\left(F \mathrm{O}^{2}\right)+(0.0398 P)^{2}+1.6972 P\right]$

WHERE $P=\left(F^{2}+2 F C^{2}\right) / 3$

$(\Delta / \sigma)_{\max }=0.001$

$\Delta \rho_{\max }=0.61$ e $\AA^{-3}$

$\Delta \rho_{\min }=-0.36$ e $\AA^{-3}$

Extinction correction: none

Extinction coefficient: - 


\section{Compound III (CCDC 1439899)}

Diffractometer operator C. Jandl

scanspeed 30 s (scan 1-4) and 5 s (scan 5) per frame

$\mathrm{dx} 40 \mathrm{~mm}$

2747 frames measured in 5 data sets

phi-scans with delta_phi $=0.5$

omega-scans with delta_omega $=0.5$

\section{Crystal data}

\begin{tabular}{|l|l|}
\hline $\mathrm{C}_{23} \mathrm{H}_{23} \mathrm{~F}_{6} \mathrm{~N}_{2} \mathrm{Zn}$ & $D_{\mathrm{x}}=1.435 \mathrm{Mg} \mathrm{m}^{-3}$ \\
\hline$M_{\mathrm{r}}=506.82$ & Melting point: ? K \\
\hline Monoclinic, $P 2_{1} / n$ & Mo Ka radiation, $\lambda=0.71073 \AA$ \\
\hline Hall symbol: -P 2yn & Cell parameters from 9961 reflections \\
\hline$a=12.3089(3) \AA$ & $\theta=2.4-26.4^{\circ}$ \\
\hline$b=9.6492(3) \AA$ & $\mu=1.11 \mathrm{~mm}^{-1}$ \\
\hline$c=19.8991(5) \AA$ & $T=200 \mathrm{~K}$ \\
\hline$\beta=96.953(1)^{\circ}$ & Fragment, yellow \\
\hline$V=2346.06(11) \AA^{3}$ & $0.33 \times 0.33 \times 0.27 \mathrm{~mm}$ \\
\hline$Z=4$ & \\
\hline$F(000)=1036$ & \\
\hline
\end{tabular}

Data collection

\begin{tabular}{|l|l|}
\hline $\begin{array}{l}\text { Bruker APEX-II CCD } \\
\text { diffractometer }\end{array}$ & 4814 independent reflections \\
\hline $\begin{array}{l}\text { Radiation source: fine-focus sealed tube } \\
\text { Triumph optic monochromator }\end{array}$ & $R_{\text {int }}=0.018$ reflections with i $>2 \sigma(\mathrm{i})$ \\
\hline Detector resolution: 16 pixels $\mathrm{mm}^{-1}$ & $\theta_{\max }=26.4^{\circ}, \theta_{\min }=2.1^{\circ}$ \\
\hline phi-and $\omega$-rotation scans & $h=-15 \quad 15$ \\
\hline $\begin{array}{l}\text { Absorption correction: multi-scan } \\
S A D A B S, \text { Bruker, } 2008 \mathrm{~b}\end{array}$ & $k=-12 \quad 11$ \\
\hline$T_{\min }=0.677, T_{\max }=0.745$ & $1=-24 \quad 24$ \\
\hline
\end{tabular}


46707 measured reflections

Refinement

Refinement on $F^{2}$

Least-squares matrix: full

$R\left[F^{2}>2 \sigma\left(F^{2}\right)\right]=0.046$

$w R\left(F^{2}\right)=0.129$

$S=1.03$

4814 reflections

312 parameters

39 restraints

- constraints

Primary atom site location: structure-invariant direct methods
Secondary atom site location: difference

Fourier map

Hydrogen site location: inferred from neighbouring sites

$\mathrm{H}$-atom parameters constrained

$\mathrm{W}=1 /\left[\Sigma^{2}\left(F \mathrm{O}^{2}\right)+(0.0563 P)^{2}+2.3228 P\right]$

WHERE $P=\left(\mathrm{FO}^{2}+2 \mathrm{FC}^{2}\right) / 3$

$(\Delta / \sigma)_{\max }=0.001$

$\Delta \rho_{\max }=0.61 \mathrm{e} \AA^{-3}$

$\Delta \rho_{\min }=-0.67 \mathrm{e} \AA^{-3}$

Extinction correction: none

Extinction coefficient: - 